\title{
Integrating Aggregate-Data and Individual-Patient-Data in Meta-Analysis: An Empirical Assessment and an Alternative Method for the Two-Stage Approach
}

\author{
Nik Ruzni Nik Idris ${ }^{1} \&$ Nurul Afiqah Misran ${ }^{1}$ \\ ${ }^{1}$ International Islamic University Malaysia, Malaysia \\ Correspondence: Nik Ruzni Nik Idris, Department of Computational and Theoretical Sciences, Kulliyyah of \\ Science, International Islamic University Malaysia, Kuantan 25200 Pahang, Malaysia Tel: 60-9570-5072. E-mail: \\ ruzni@iium.edu.my
}

Received: September 2, 2015

Accepted: September 25, 2015 Online Published: November 30, 2015

doi:10.5539/mas.v9n13p237

URL: http://dx.doi.org/10.5539/mas.v9n13p237

This research is supported by the Fundamental Research Grant Scheme (FRGS 13-093-033), Ministry of Higher Education, Government of Malaysia.

\begin{abstract}
In this study, we compared the efficacy of the overall meta-analysis estimates that used only the available aggregate data (AD) studies against those that combined the available AD and individual patient data (IPD) studies. We introduced some modifications to the existing two-stage method for combining the AD and IPD studies. We evaluated the effects of these modifications on the estimates of the overall treatment effect, and investigated the influence of the number of studies included in the meta-analysis, N, and the ratio of AD: IPD on these estimates. We used percentage relative bias (PRB), root mean-square-error (RMSE), and coverage probability to assess the overall efficiency of these estimates. The results revealed the superiority of estimates from the combined AD: IPD studies over those that utilized only the available AD in terms of both the accuracy and the RMSE. We found that the current method for combining the AD:IPD studies provided poor coverage probabilityand that the proposed methods generated improved coverage probability by more than $40 \%$ while maintaining the level of bias and RMSE at par to their existing counterparts. These findings validated the importance of utilizing both the AD and IPD studies whenever they are available, and demonstrated the significance of proper technique for combining these studies in order to obtain better overall estimates.
\end{abstract}

Keywords: aggregate data, bias, individual patient data, meta-analysis, RMSE, simulation

\section{Introduction}

Meta-analysis is a statistical technique for integrating quantitative results from several independent sources. Theoretically, a meta-analysis should provide more reliable results than that of an individual study, as a meta-analysis is based on the entire body of research. A traditional meta-analysis involves the integration of aggregate data $(\mathrm{AD})$, which is extracted from the individual study publications. Examples of AD include a standardized mean difference for continuous outcomes or the number of events and participants for binary outcomes. The weighted average of the effects across the studies is taken as the overall treatment effect. Methods such as the inverse variance method (DerSimonian \& Laird, 1986), for continuous data, or the Mantel-Haenszel method (Mantel \& Haenszel, 1959), for binary data, may be utilized to obtain the overall estimate. A meta-analysis may alternatively be performed based on individual patient data (IPD), in which case raw data from an individual study are obtained and synthesized directly. In this case, a methodology adopted for analysis of primary data may be applied in estimating the overall effect. IPD meta-analysis has several advantages compared to AD meta-analysis, particularly in terms of type of analyses that can be performed. However, IPD meta-analysis is usually more costly and time consuming (Steward \&Tierney, 2002, Simmonds et al.,2005, Jones et al., 2009), as well as seldom available from each of the individual studies. Another approach which is gaining in popularity is the utilizations of both the available AD and IPD, known as mixed data (MD), in a meta-analysis. Combining available IPD with the AD maximizes the utilisation of available information, as it allows for a larger number of patients, hence a greater part of the evidence-based could be included. (Jones et al., 2009; Cooper \& 
Patall, 2009, Donegan et al., 2013).

Literature on the comparison of AD and IPD meta-analysis estimates has been quite extensive (Jones et al.,2009, Lambert et al.,2001). However, the researchers for only two studies had examined the efficacy of the estimates that are based on combined-level studies (Riley et al., 2008, Idris \&Abdullah, 2015). Riley et al. (2008) compared estimates obtained from a meta-analysis that combined IPD and AD studies using a two-stage method to those using all-AD studies. Riley et al. (2008) took their data from a study on the effects of hypertension (Wang et al., 2005), and compared their results with those of the all-IPD estimates. Riley et al. (2008) results showed that the benefit of combining the IPD with AD increases as the proportion of IPD studies decreases, and that the differences in the estimates from all AD and AD: IPD combined studies were relatively small. These findings are supported by another simulation-based study (Idris \& Abdullah, 2015), for which the researchers concluded that the benefit of combining the data is greater if the majority of the studies to be combined are at AD-level and that if more than $80 \%$ of the studies are IPD, including the $\mathrm{AD}$ is not recommended as the statistical benefit is not significant. In fact, in this case, it would only serve to increase the overall SE. Additionally, Idris and Abdullah (2015) found that eventhough combined data produced better estimates compared to those from AD-only studies, the bias introduced into the overall estimates were rather high, particularly when the majority of the combined studies were at the AD level. Donegan et al (2013) examined the benefit of combining IPD and AD using treatment-by-covariate interactions in the model, when assessing the underlying consistency assumption. They found that using the combined data, they were able to identify statistically significant regression coefficients for the interactions better compared to that of using IPD alone and $\mathrm{AD}$ alone.

Currently four methods, based on one-stage and two-stage approaches, are available for combining the IPD and AD, namely, the standard two-stage method (Collete et al., 1998, Tudor et al., 2001), partially reconstructed IPD method (Turner et al., 2000, Whitehead et al., 2001), multi-level modeling method (Goldstein et al., 2000) and the hierarchical related regression using a Bayesian framework (Jackson et al., 2006). The standard two-stage method uses a two-stage approach whereas the other three methods are based on one-stage approach. (Riley et al., 2007, Donegan et al., 2013) found that of 33 meta-analyses that combined the AD and IPD studies, 29 used a standard two-stage method and the other four have adopted the reconstruction method for binary outcome data. None of the researchers for the meta-analyses reviewed used either multi-level models or the Bayesian approach. The popularity of a two-staged approach may be attributed to its practical simplicity and ease of understandig without overwhelming the readers with heavy theoretical background information.

Although the two-stage method has been widely accepted in practice, discussion on the methodology of this approach and its implications on the overall estimates is limited. The assessments of the statistical properties of these estimates has not been fully investigated. In this study, we examined how the estimate of overall treatment effect from combined AD: IPD studies compared to those from conventional AD-only meta-analyses, in cases where both levels of data were available. We conducted a simulation study to quantify and compare the percentage relative bias (PRB) and the root mean-square-error (RMSE) of meta-analysis estimates extracted from AD-only studies and from selected ratios of AD: IPD studies. Further, we explored the possibility of improving the statistical properties of the overall estimates with some modifications to the existing two-stage method. We proposed two modifications to the existing two-stage approach. The first modification involved the utilization of the number of studies as the weightage in combining the overall estimates, and the second modification involved utilizing the grouped-variance from the two levels of data as the weightage. We evaluated the effects of these modifications to the estimates of the overall treatment effect and compared them to those using the existing method. We used the PRB and RMSE to assess the overall efficiency of these estimates. Additionally, we investigated the influence of the ratio of AD: IPD, as well as the effects of the number of studies included in the meta-analysis, $\mathrm{N}$, on the accuracy and precision of the overall treatment effects estimates.

\section{Method}

\subsection{Generation of Data}

We used a simulation approach to generate IPD level response, denoted as $y_{i j}$, representing a response from patient $j$ from study $i$, where

$$
y_{i j}=\beta_{0 i}+\beta_{1 i} t_{i j}+\epsilon_{i j}
$$

$\beta_{0 i}$ was the random study effect, which can be written as $\beta_{0 i}=\beta_{0}+\nu_{0 i}$, where $\nu_{0 i}$ is the random term which is assumed to be normally distributed with variance $\sigma_{\nu_{0 i}}^{2}, \nu_{0 i} \sim N\left(0, \sigma_{\nu_{0 i}}^{2}\right)$, thus $\beta_{0 i} \sim N\left(\beta_{0}, \sigma_{\nu_{0}}^{2}\right)$. $t_{i j}$ represented a dummy covariate for treatment which took two values, namely 0 for the control and 1 for the 
treatment arm, $\beta_{1 i}$ was the random treatment effect, where $\beta_{1 i}=\beta_{1}+\nu_{1 i}$, and $\nu_{1 i}$ was the random effect term, assumed to be normally distributed with between study treatment variance of $\tau^{2}, \nu_{1 i} \sim N\left(0, \tau^{2}\right)$ thus $\beta_{1 i} \sim N\left(\beta_{1}, \tau^{2}\right)$. Thus $\tau^{2}$ represents the unexplained heterogeneity in treatment effect across studies. $\epsilon_{i j}$ were the sampling random error terms for the response from patient $j$ within study $i$, given by $\epsilon_{i j} \sim N\left(0, \sigma_{\epsilon}^{2}\right)$. We assumed $\beta_{0 i}, \beta_{1 i}$ and $\epsilon_{i j}$ were independent and for simplicity, we assumed each study had an equal number of patients in each treatment arm (i.e $n_{0 i}=n_{1 i}=1 / 2 n_{i}$ for $i=1,2, \ldots, N$ ). We had adapted earlier study (Idris $\&$ Abdullah, 2015) and modified it to create a more heterogeneous meta-analysis data, by arbitrarily assigning the following values to the fixed effects: $\beta_{0}=0$ and $\beta_{1}=7$, which was arbitrarily chosen as a common size of treatment effect within observational meta-analysis (Robertson et al., 2004). For the random effects, we assigned the following values to create a moderately heterogeneous effect ( I: $25 \%$ to $50 \%$ ); $\sigma_{\nu_{0}}^{2}=1$ and $\tau^{2}=2$ while we allowed the $\sigma_{\epsilon}^{2}$ to vary randomly between 1 to 25 . These values were chosen to allow for the required heterogeneity level among the effects sizes. We created the AD by taking the differences of the means of each treatment arm in each individual study, and created the combined AD: IPD data by selecting a certain ratio of AD and IPD studies generated earlier, as detailed in the proceeding paragraph.

We denoted an "AD-only" meta-analysis when utilizing only the available AD studies in a case where both $\mathrm{AD}$ and IPD studies were available for a meta- analysis. We used the term "all-AD" and "all-IPD" to describe studies that were used when all of the studies available for meta-analysis were AD and IPD, respectively.

The factors that we varied in this simulation study were the number of studies included in the meta- analysis, $\mathrm{N}$, ( $N=10,20,30$ and 90) and the ratio of AD: IPD studies in a meta-analysis, namely, 0:100, 20:80, 30:70, 40:60, 50:50, 60:40, 80:20 and 100:0, while the size of the samples, $n$, were fixed at 60, a moderate sample size (Idris and Robertson, 2009). We combined each ratio of AD: IPD using three methods; namely the existing standard two-stage method (SM), and two modifications of existing methods, namely, the N-weighted estimates (NW), and the inverse group-variance weighted estimates (IGVW). All the parameters in this paper can be estimated using restricted maximum likelihood (REML) within the suitable packages from $\mathrm{R}$ statistical analysis software (R Dev., 2008) for mixed models such as the Linear Mixed Effect (LME) or the Non-Linear Mixed Effect (NLME).

\subsection{Assessment of the Estimates}

These specifications generated 32 sets of meta-analyses comprising four all-IPD studies, four all-AD studies and 24 sets of AD: IPD studies consisting of different combinations of $N$ and AD: IPD ratios. We obtained the overall estimates for the IPD studies using the usual mixed-effect model, and applied the standard inverse-variance weighted method to obtain estimates for the AD studies. We combined the AD: IPD studies using the three methods under consideration, namely, SM, NW and IGVW and computed the overall estimates accordingly. We replicated each meta-analysis 1,000 times and for each replication we computed the overall estimate of the treatment effects, PRB, RMSE and their corresponding standard error (SE). We used the means of these 1000 replications for the analysis. We also recorded the coverage probability at $95 \%$ nominal value. We used percentage relative bias, root mean-square-error, and the coverage probability to assess the overall efficiency of these estimates (Idris \& Abdullah, 2015)

\subsubsection{Percentage Relative Bias (PRB)}

We computed the PRB as the percentage difference between the true treatment effect and the estimated treatment effect. The mean PRB was given by:

$$
\text { mean } \operatorname{PRB}(\hat{\theta})=\frac{\sum_{t=1}^{K}\left(\theta-\hat{\theta}_{t}\right) / \theta}{K} \times 100 \%
$$

where $\hat{\theta}_{t}$ was the estimate of the treatment effect from simulation $t, \theta$ was the true treatment effect, and $K$ was the number of simulations.

\subsubsection{Root Mean Square Error (RMSE)}

The RMSE is the square root of the mean-square-error (MSE) for the overall estimate of treatment effect, where the mean MSE over $K$ simulations was given by 


$$
\text { mean MSE }=\frac{\sum_{t=1}^{K}\left[\operatorname{bias}\left(\hat{\theta}_{t}\right)^{2}+S E\left(\hat{\theta}_{t}\right)^{2}\right]}{K}
$$

where $\operatorname{bias}\left(\hat{\theta}_{t}\right)$ was the observed bias for $\hat{\theta}_{t}$ and $S E\left(\hat{\theta}_{t}\right)$ was the standard error corresponding to the overall estimate from meta-analysis at simulation number $t$.

\subsubsection{Coverage Probability}

We estimated the coverage probability by taking the proportion of the number of times the estimated $95 \%$ confidence interval included the true value of $\theta$ out of the total number of simulations $K$.

\subsection{Two-Stage Method for Combining the AD and IPD}

\subsubsection{Standard Two-Stage Method (SM)}

Suppose there was $N=N_{1}+N_{2}$ studies where $N_{1}$ was the number of AD level studies with effects estimates $\hat{\theta}_{1}, \hat{\theta}_{2}, \ldots, \hat{\theta}_{N 1}$ and corresponding variances given by $V\left(\hat{\theta}_{1}\right), V\left(\hat{\theta}_{2}\right), \ldots, V\left(\hat{\theta}_{N 1}\right)$ while $N_{2}$ was the number of IPD level studies. In a standard two-stage method, the available IPD are first reduced to AD in each study before they are combined with the existing $\mathrm{AD}$ studies using standard meta-analysis of $\mathrm{AD}$ techniques. Suppose $\hat{\theta}_{1}^{*}, \hat{\theta}_{,}^{*}, \ldots, \hat{\theta}_{N \text {, }}^{*}$ denoted the study specific effects from the reduced IPD studies, with corresponding variances $V\left(\hat{\theta}_{1}^{*}\right), V\left(\hat{\theta}_{2}^{*}\right), \ldots, V\left(\hat{\theta}_{N 1}^{*}\right)$. The effect $\hat{\theta}_{i}^{*}$ for instance may represent the difference in the mean response of individual patients for treatment and control arms from study $i$ and the $V\left(\hat{\theta}_{i}^{*}\right)$ represents the pooled treatment and control arms variances corresponding to their respective effects for study $i$.

We computed the overall effect using the standard inverse variance weighted method given by

$$
\hat{\theta}_{\text {overall }}=\frac{\sum w_{i} \hat{\theta}_{i}+\sum w_{i}^{*} \hat{\theta}_{i}^{*}}{\sum w_{i}+\sum w_{i}^{*}}
$$

where $w_{i}$ and $w_{i}^{*}$ are the weights given by $w_{i}=1 / V\left(\hat{\theta}_{i}\right)$ and $w_{i}^{*}=1 / V\left(\hat{\theta}_{i}^{*}\right)$.

\subsubsection{Modifications of SM}

In this modification, we computed the overall effects from $\mathrm{AD}$ and the overall effects from IPD separately. These two effects were combined using the weighted average, where the weights were (1) the number of studies in each group, that is the $\mathrm{N}$-weighted estimate (NW), and (2) the inverse variances from the AD and IPD group, that is, the inverse group-variance weighted estimate (IGVW).

\subsubsection{N-Weighted Estimate (NW)}

Using the same notation as in the preceding section, we let $N_{1}$ and $N_{2}$ be the number of AD and IPD studies, respectively, in a given meta-analysis. We first computed the overall estimate for AD studies, denoted $\hat{\theta}_{A D}$, using standard AD meta-analysis method (Brockwell \& Gordon, 2001). The overall estimate for IPD studies, $\hat{\theta}_{I P D}$, were obtained directly using the mixed effect model (interested readers may refer to Whitehead et al.,2001) without reducing them to the AD level first. In this case, the $\hat{\theta}_{I P D}, \tau^{2}, \sigma_{\epsilon}^{2}$, and $\sigma_{\nu_{0 i}}^{2}$ were estimated simultaneously. We then combined these two estimates using the $N_{1}$ and $N_{2}$ as the weights, as follows:

$$
\hat{\theta}_{\text {all } 1}=\frac{(N 1) \hat{\theta}_{A D}+(N 2) \hat{\theta}_{I P D}}{N 1+N 2}
$$

The variance of the overall estimate, $V\left(\hat{\theta}_{\text {all } 1}\right)$, was the pooled estimate of the variances of the two groups.

\subsubsection{Inverse Group-Variance Weighted Estimate (IGVW)}

In the second modification, we utilized the inverse variances of each group as the weights. For the AD studies, the weight, $w_{A D}$, was simply the inverse of the overall variance for the AD group, $w_{A D}=1 / V\left(\hat{\theta}_{A D}\right)$, for IPD studies, the weight, $w_{I P D}$ was the inverse variance for IPD group $w_{I P D}=1 / V\left(\hat{\theta}_{I P D}\right)$, and the overall estimate was given by

$$
\hat{\theta}_{a l l 2}=\frac{\left(w_{A D}\right) \hat{\theta}_{A D}+\left(w_{I P D}\right) \hat{\theta}_{I P D}}{w_{A D}+w_{I P D}}
$$

The variance of the overall estimate, as in the case of standard meta-analysis, was given by

$$
V\left(\hat{\theta}_{\text {all }}\right)=\frac{1}{w_{A D}+w_{I P D}}
$$




\section{Results}

3.1 The PRB, RMSE and the Coverage Probability of Estimates from Meta-Analysis with AD-Only Studies and Combined AD: IPD Studies

Figure 1 presents the distributions of PRB, RMSE and the coverage probability of estimates from AD-only and the combined AD:IPD meta-analysis for the selected range of $N(N=10,20,30,90)$ and six combinations of AD:IPD. Clearly, the AD:IPD meta-analysis generated lower bias with the mean PRB ranges from $1.5 \%$ to $5.0 \%$ compared to the conventional $\mathrm{AD}$-only meta-analysis (mean PRB $=-11 \%$ to $5.3 \%$ ). The majority of PRB from $\mathrm{AD}$-only studies were negative for the percentage of $\mathrm{AD}$ within the combined studies that were less than $40 \%$, suggesting overestimated effects. On the other hand, the estimates of treatment effects from the AD:IPD studies remained positive suggesting some underestimation in the estimates although not as severe as those of the AD-only in terms of the magnitude of the bias.

As in the case of PRB, the distribution of the RMSE for estimates from AD-only and combined AD:IPD meta-analysis showed the latter were lower, averaging from 0.53 to 1.22 compared to those from AD-only (mean RMSE of 0.55 to 2.17). The RMSE decreased as the number of studies, N, increased, which was expected as the $\mathrm{SE}$ tended to decrease with increasing $\mathrm{N}$.

The coverage probability was better for estimates with AD-only studies compared to those from the combined studies. For nominal values of $95 \%$, the coverage ranges from $70 \%$ to $90 \%$ for $\mathrm{AD}$-only studies. The coverage for combined AD: IPD studies was slightly lower, ranging from $50 \%$ to $70 \%$, increasing with the proportion of $\mathrm{AD}$ within the AD: IPD ratio.

\subsection{Comparison of PRB and RMSE of the Existing Method(SM) Against the Proposed Methods (NW and IGVW) for Combining the $A D$ and IPD}

A comparison of the observed statistical properties of estimates from the existing method for combining the AD: IPD studies and the proposed modifications suggested possible benefit of the latter. The trend of PRB from the three methods was quite similar (see Figure 2). We noted that the proposed methods, NW and IGVW, displayed smaller PRB than the existing method when the number of studies included in the meta-analysis was at moderate range, $\mathrm{N}(20<\mathrm{N}<60)$. Of the two proposed methods, IGVW consistently produced smaller PRB than the NW for all the ratios of AD: IPD considered.

Figure 3 shows the RMSE was slightly lower when the existing method, SM, was used to combine the AD and IPD studies when a majority of the studies within the AD: IPD studies were IPD. Of the two proposed methods, IGVW showed a smaller RMSE compared to NW. This trend was similar for all ratios of AD: IPD, and as the proportions of AD increased, the RMSE of the estimates from all three methods converged to the same value.

\subsection{The Coverage Probability and SE of Estimates Based on Studies with SM, NW and IGVW}

The proposed methods provided estimates with better coverage than those from the existing method. NW provided mean coverage of approximately $90 \%$ for a nominal value of $95 \%$ whereas the corresponding mean coverage for IGVW was approximately $70 \%$ against an average of $50 \%$ using the existing method (see Figure 4). We noted that the SE from estimates based on the standard method were underestimated, resulting in a confidence band which was too narrow. (see Table 1). The best coverage was given by NW, which may be attributed to its relatively larger and more realistic SE then both the SM and the IGVW, despite a larger bias.

\subsection{The Effects of Ratio of AD: IPD and the Number of Studies, $N$, on the Overall Estimates}

The number of studies included in a meta-analysis has some effects on the accuracy of the overall estimate. Figure 2 exhibits an increasing trend in PRB as the number of studies increases, which is particularly apparent in the two proposed methods. The PRB was expected to generally increase with $\mathrm{N}$ due to the effects of weightage used in computation of the overall estimates where the weights were generally larger for smaller study-variance (i.e larger $\mathrm{N})$. However, for meta-analysis with a moderate number of studies $(\mathrm{N}<30)$, this effect was minimal. We noted that the effect of AD: IPD on the bias was relatively small. However, the differences in the bias in all three methods, across $\mathrm{N}$, reduced as proportions of $\mathrm{AD}$ within the AD:IPD ratios increased, implying that the proposed NW and IGVW methods did not have much of an advantage over SM in terms of bias, when the majority of the studies within the combined AD:IPD were at AD level.

Figure 3 shows that as $\mathrm{N}$ increased, the RMSE decreased, as expected, due to lower SE for a larger number of studies included in a meta-analysis (see Table 1). As expected, the SE reduced as $\mathrm{N}$ increased and relatively smaller SEs were observed from the SM approach. Figure 3 additionally illustrated that the RMSE of the estimates from all three methods converged for large $\mathrm{N}$, suggesting minimal beneficial effects of utilizing the 
proposed methods in terms of RMSE when $\mathrm{N}$ was large $(\mathrm{N}>40)$. In terms of the effects of the AD:IPD ratio, we noted that the RMSE reduced slightly as the proportion of AD increased, again reflecting the effects of SE which were lower in AD compared to those from IPD studies. The RMSE from the three methods appeared to close up as the proportion of $\mathrm{AD}$ increased. The number of studies, N, and the ratios of AD: IPD did not have notable effects on the coverage probability (see Figure 4). This trend were observed on all three methods.
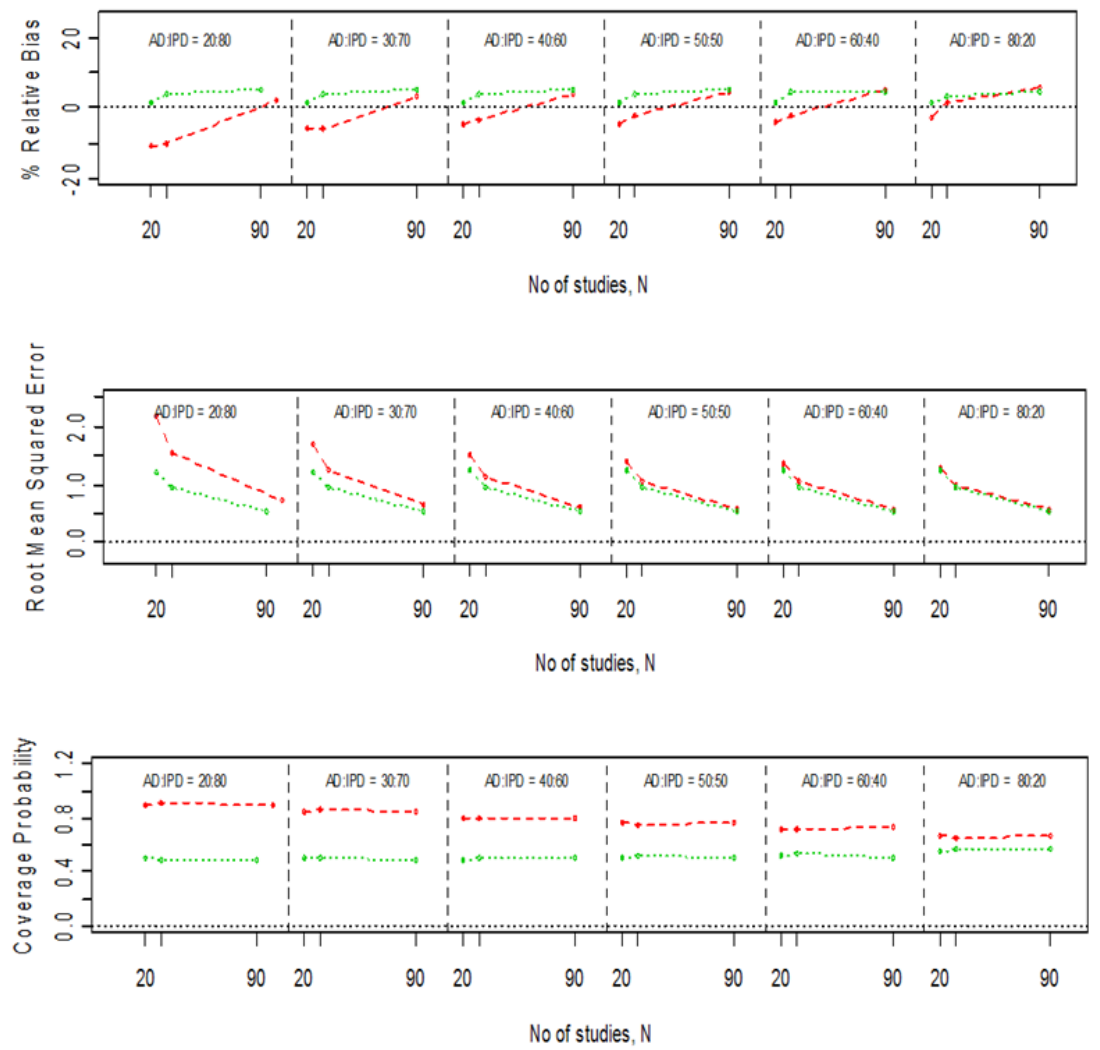

Figure 1. Distribution of PRB, RMSE and the Coverage probability for the overall estimates from AD-only and AD:IPD studies. Legend: RED - AD-only studies; GREEN - Combined AD: IPD studies using the standard method (SM)
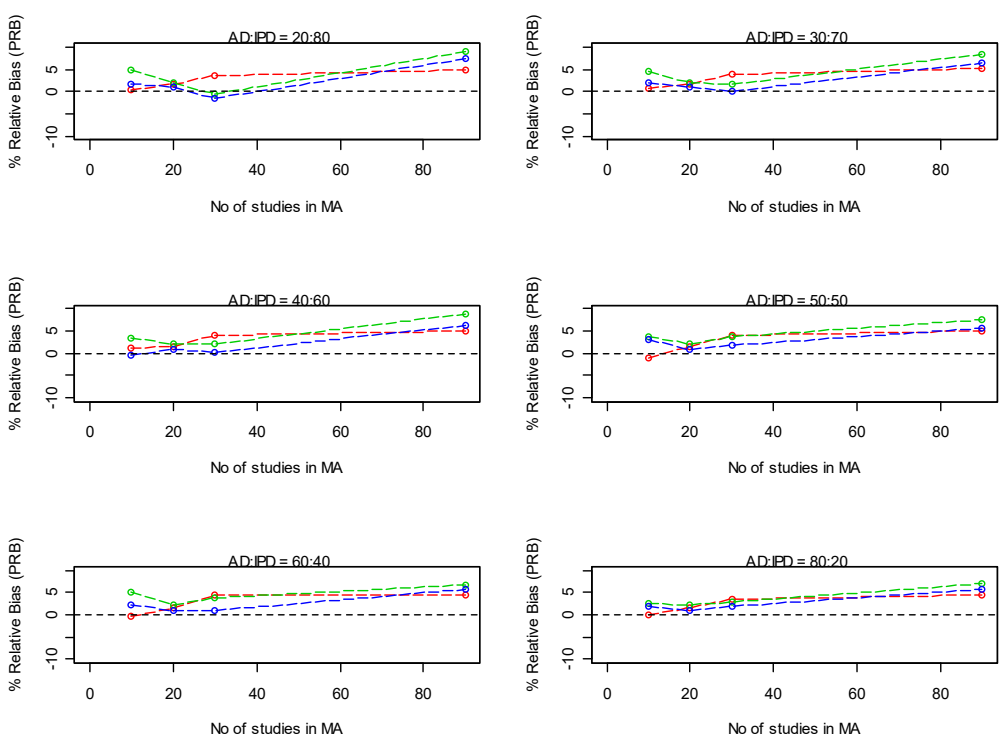

Figure 2. Distribution of PRB for the overall estimates using SM, NW and IGVW methods for the selected range of $\mathrm{N}$ ( $\mathrm{N}$ from 10 to 90 ) and six combinations of AD: IPD ratios. Legend: Red: existing SM; Green: NW; Blue: 
IGVW
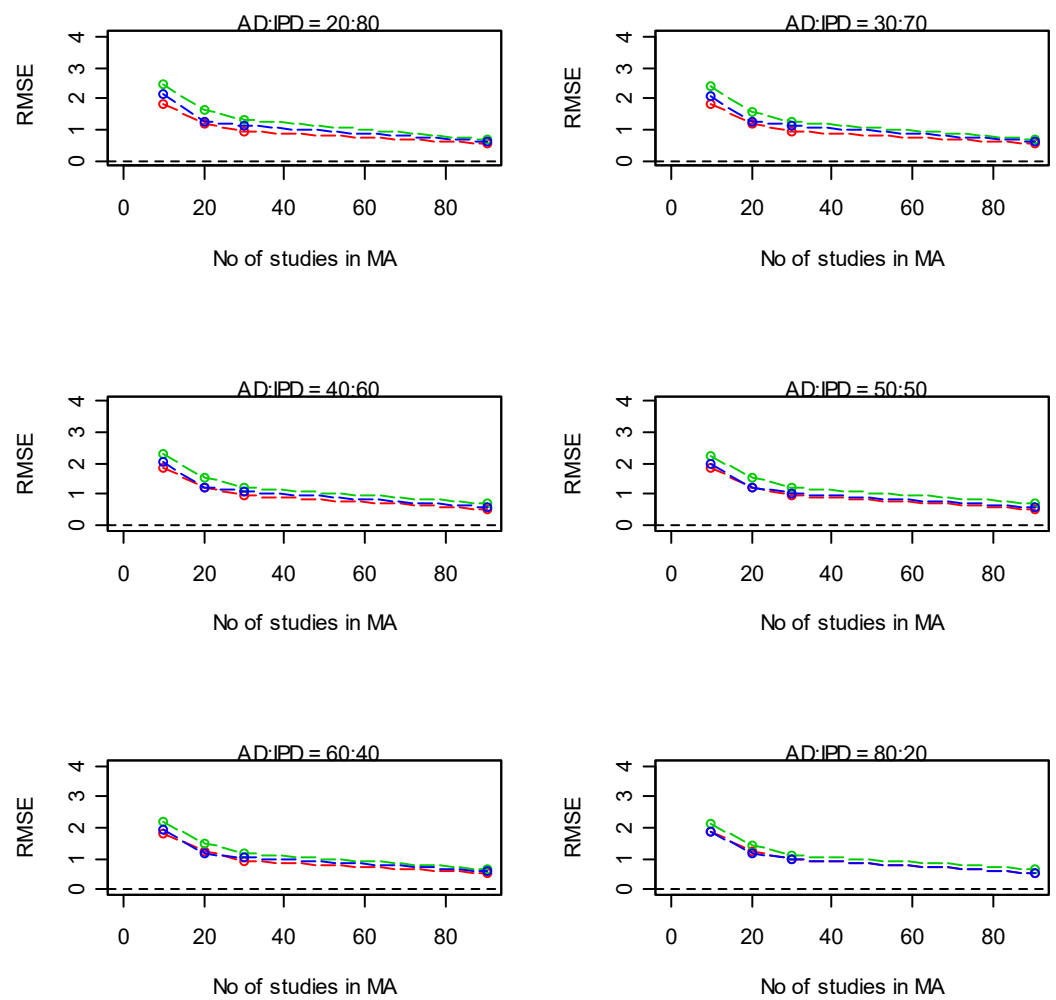

Figure 3. Distribution of RMSE for the overall estimates using SM, NW and IGVW methods for six combinations of AD:IPD and ranges of N from 10 to 90. Legend: Red: existing SM; Green: NW; Blue:IGVW
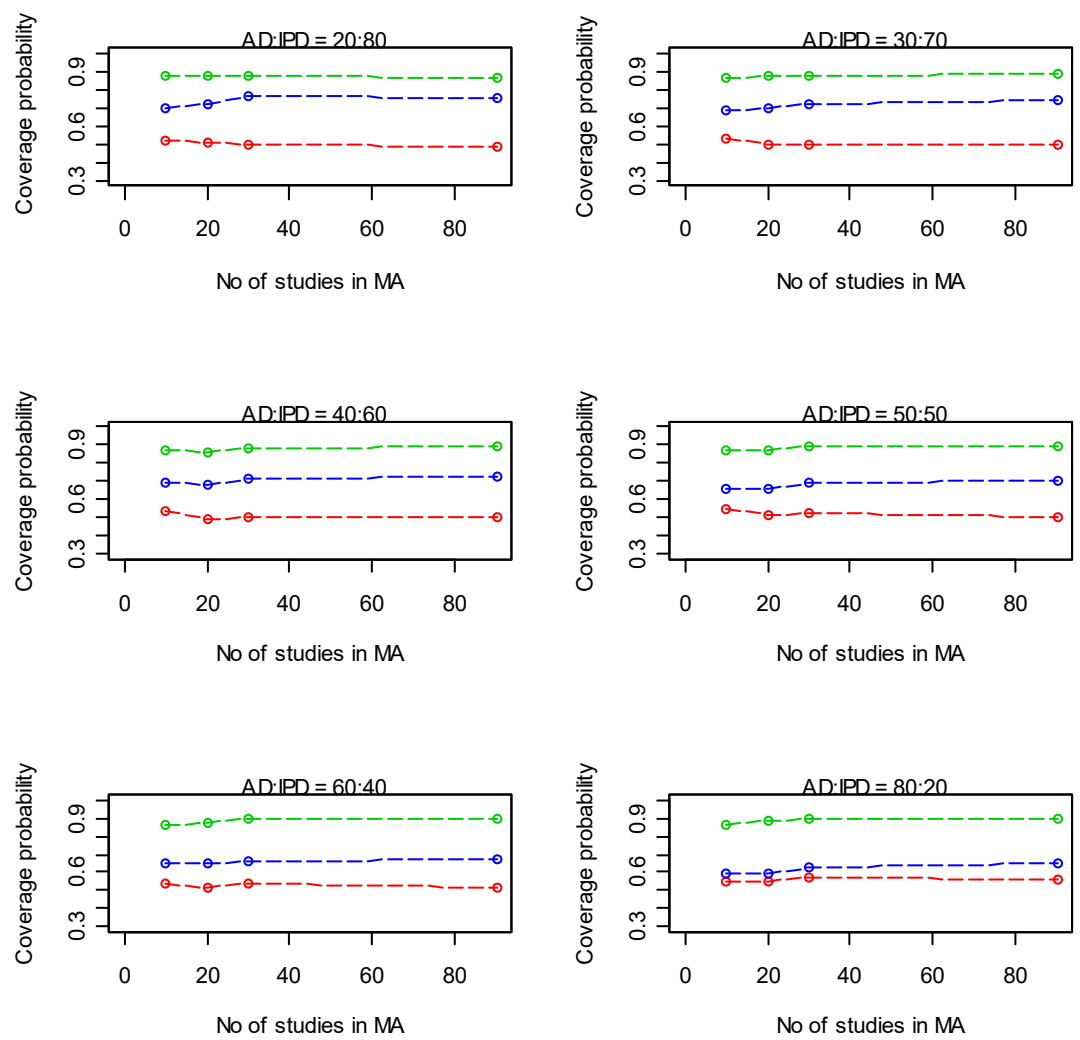

Figure 4. Distribution of coverage for the overall estimates using SM, NW and IGVW methods for six combinations of AD:IPD and ranges of $\mathrm{N}$ from 10 to 90. Legend: Red: existing SM; Green:NWE; Blue:IGVW 
Table 1. Distribution of the estimates of overall treatment effects (ES) and their corresponding SE

\begin{tabular}{ccccl}
\hline N & AD:IPD & $\begin{array}{c}\text { SM } \\
\text { ES (SE) }\end{array}$ & $\begin{array}{c}\text { NW (SE) } \\
\text { ES }\end{array}$ & $\begin{array}{c}\text { IGVW } \\
\text { ES (SE) }\end{array}$ \\
\hline 10 & $20: 80$ & $6.97(1.32)$ & $6.66(1.95)$ & $6.88(1.60)$ \\
& $40: 60$ & $6.91(1.33)$ & $6.76(1.81)$ & $7.04(1.50$ \\
& $50: 50$ & $7.06(1.33)$ & $6.74(1.75)$ & $6.78(1.45)$ \\
& $60: 40$ & $7.02(1.33)$ & $6.65(1.70)$ & $6.85(1.42)$ \\
& $80: 20$ & $6.99(1.34)$ & $6.83(1.65)$ & $6.87(1.38)$ \\
20 & $20: 80$ & $6.89(0.89)$ & $6.86(1.29)$ & $6.92(1.10)$ \\
& $40: 60$ & $6.89(0.89)$ & $6.87(1.22)$ & $6.93(1.02)$ \\
& $50: 50$ & $6.89(0.90)$ & $6.85(1.19)$ & $6.92(0.99)$ \\
& $60: 40$ & $6.88(0.89)$ & $6.86(1.17)$ & $6.91(0.97)$ \\
& $80: 20$ & $6.89(0.90)$ & $6.89(1.14)$ & $6.92(0.94)$ \\
30 & $20: 80$ & $6.75(0.70)$ & $7.04(1.01)$ & $7.09(0.89)$ \\
& $40: 60$ & $6.72(0.69)$ & $6.89(0.96)$ & $6.99(0.86)$ \\
& $50: 50$ & $6.72(0.69)$ & $6.85(0.93)$ & $6.87(0.79)$ \\
& $60: 40$ & $6.68(0.69)$ & $6.73(0.93)$ & $6.92(0.77)$ \\
& $80: 20$ & $6.76(0.70)$ & $6.79(0.90)$ & $6.87(0.76)$ \\
90 & $20: 80$ & $6.64(0.39)$ & $6.37(0.55)$ & $6.46(0.47)$ \\
& $40: 60$ & $6.65(0.39)$ & $6.38(0.53)$ & $6.55(0.44)$ \\
& $50: 50$ & $6.66(0.40)$ & $6.47(0.53)$ & $6.59(0.43)$ \\
& $60: 40$ & $6.68(0.40)$ & $6.53(0.52)$ & $6.60(0.43)$ \\
& $80: 20$ & $6.69(0.40)$ & $6.52(0.51)$ & $6.60(0.41)$ \\
\hline
\end{tabular}

Note: The assigned value of ES, $\beta_{1}=7 . \mathrm{SM}=$ the existing method of combining the AD and IPD. NWE=the modification based on $\mathrm{N}$. IGVW=the modification based on grouped-variance.

\section{Discussion}

Our simulation results revealed clear evidence of the superiority of estimates from the combined AD: IPD studies over those that utilized only the available AD, in terms of both the accuracy and the overall RMSE. These findings were consistent with earlier results (Riley et al., 2008, Idris \& Abdullah, 2015) in which they compared the estimates from all-IPD and all-AD studies to the combined AD: IPD studies. We demonstrated that the value of combining AD: IPD was significant even if only $20 \%$ of the available studies were at the IPD level. The only setback for the combined level studies was that its coverage probability was relatively low compared to the AD-only meta-analysis, when the existing method for combining was used.

We postulated that the coverage probability may be improved if we introduced some modifications to the existing methodology that was used to combined the AD: IPD studies. We considered grouped-based weightage that utilized all available information contained in the IPD studies. We demonstrated that in cases in which the majority of the available studies within the AD:IPD were in AD form, the proposed methods were preferable over the existing method of combining as they produced estimates with higher coverage probability and lower PRB and RMSE, in some cases. The proposed methods provided better statistical properties than the existing method when the number of studies in a meta-analysis, $N$, was moderate $(10<N<60)$. For larger $N(N>60)$, the benefit of the proposed method remain relatively good in terms of coverage, but was not markedly different in terms of bias and RMSE than those from the existing method.

Evidently the greatest advantage of the proposed method of combining the AD: IPD was in terms of the coverage probability. This study revealed that the current method used to combined the AD: IPD studies provided poor coverage. We noted that this situation may be attributed to underestimation of the SE of estimates produced using the existing method, which in turn, produced an interval which was narrower than it should have been. Of the two modifications introduced, NW, which utilized the number of studies, N, as the weightage, and IGVW, which utilized the inverse group-variances as the weightage, the latter appeared to offer consistently better performance in terms of bias and RMSE. IGVW however, displayed lower coverage probability than NW, albeit better than the existing method. In both modification methods, estimates from IPD studies were estimated directly, without reducing them to AD level first, thus utilizing all the information available within the IPD.

We acknowledge the lack of theoretical support for the proposed modifications of the two-stage methods due to their complex analytical approach. Nonetheless, it was a simple modification, in which we evaluated the AD and 
IPD separately and combined them using the typical weights, which was simpler to implement and easier to interpret. Our simulation results confirmed that these modifications yielded estimates with improved coverage probability, albeit it should be interpreted with caution as results may apply only to the data characteristics under investigation.

One of the main goals of meta-analysis is to draw general inferences about the research problem from the overall estimate based on a collection of independent studies. Thus a reliable overall estimate is crucial in meta-analysis. In this article we confirmed that combining the available AD and IPD provided more reliable overall estimates and better statistical properties. Therefore, we strongly recommend that, whenever possible, practitioners should include the available IPD when performing conventional meta-analysis. Another important finding suggested that the existing method currently used to combine the AD and IPD resulted in a coverage probability which was generally too low. This information was imperative in light of recent review of current practice (Riley et al., 2007), which found that $80 \%$ of meta-analyses that combined AD and IPD had used the existing two-stage method. The results of this study should provide a useful insight and may serve as a guide for practitioners when performing meta-analysis

\section{Acknowledgments}

This work was supported by the Fundamental Research Grant Scheme (FRGS 13-093-033), Ministry of Higher Education, Government of Malaysia

\section{References}

Brockwell, S. E., \& Gordon, I. R. (2001). A comparison of statistical method for meta-analysis. Stat Med, 20, 825-840. http://dx.doi.org/10.1002/sim.650

Collette, L., Suciu, S., Bijnens, L., \& Sylvester, R. (1998). Including literature data in individual patient data meta-analyses for time-to-event endpoints. Personal communication, based on abstract from the first Symposium on Systematic Reviews: beyond the basics, Oxford. Retrieved from https://lra.le.ac.uk/bitstream/2381/1388/3/mon219.pdf.txt

Cooper, H., \& Patall, E. A. (2009). The relative benefits of meta-analysis conducted with individual participant data versus aggregated data. Psychol Methods, 14(2), 165-176. http://dx.doi.org/10.1037/a0015565

DerSimonian, R., \& Laird, N. (1986). Meta-analysis in clinical trials. Control Clin Trials, 7, 177-188. Retrieved from http://www.ncbi.nlm.nih.gov/pubmed/3802833

Donegan, S., Williamson, P., D'Alessandro, U., Garner, P., \& Smith, C. T. (2013). Combining individual patient data and aggregate data in mixed treatment comparison meta-analysis: Individual patient data may be beneficial if only for a subset of trials. Stat Med., 32(6), 914-30. http://dx.doi.org/10.1002/sim.5584

Goldstein, H., Yang, M., Omar, R. Z., Turner, R. M., \& Thompson, S. G. (2000). Meta-analysis using multilevel models with an application to the study of class size effects. Appl Stat, 49, 399-412. Retrieved from http:/www.bristol.ac.uk/media-library/sites/cmm/migrated/documents/meta-analysis-class-size.pdf

Idris, N. R. N., \& Abdullah, M. H. (2015). A study on the effects of different levels of data on the overall meta-analysis estimates. Far East Journal of Mathematical Science, 96(1), $73-86$. http://dx.doi.org/10.17654/FJMSJan2015_073_086

Jackson, C., Best, N., \& Richardson, S. (2006). Improving ecological inference using individual-level data. Stat Med, 25, 2136-2159. http://dx.doi.org/10.1002/sim.2370

Jones, A. P., Riley, R. D., Williamson, P. R., \& Whitehead, A. (2009). Meta-analysis of individual patient data versus aggregate data from longitudinal clinical trials. Clin Trials, 6(1), 16-27. http://dx.doi.org/10.1177/1740774508100984

Lambert, P. C., Sutton, A. J., Abrams, K. R., \& Jones, R. D. (2001). A comparison of summary patient-level covariates in meta-regression with individual patient data meta-analysis. Journal of Clinical Epidemiology, 55, 86-94. http://dx.doi.org/10.1016/S0895-4356(01)00414-0

Mantel, N., \& Haenszel, W. (1959). Statistical aspects of the analysis of data from retrospective studies of disease. J Natl Cancer Inst, 22, 719-748. http://dx.doi.org/10.1093/jnci/22.4.719

R Development Core Team (2008). R: A language and environment for statistical computing. R Foundation for Statistical Computing, Vienna, Austria. ISBN 3-900051-07-0. Retrieved from http://www.R-project.org/

Riley, R. D., Lambert, P. C., Staessen, J. A., Wang, J, Gueyffier, F., Thijs, L., \& Boutitie, F. (2008). Meta-analysis of continuous outcomes combining individual patient data and aggregate data, Stat. Med., 27, 
1870-1893. http://dx.doi.org/10.1002/sim.3165

Riley, R. D., Simmond, M. C., \& Look, M. P. (2007). Evidence synthesis combining individual patient data and aggregate data:a systematic review identified current practice and possible methods. Journal of Clinical Epidemiology, 60, 431-439. Retrieved from http://www.ncbi.nlm.nih.gov/pubmed/17419953

Robertson, C., Boyle, P., \& Idris, N. R. N. (2004). Beyond classical meta-analysis: can inadequately reported $\begin{array}{lllll}\text { studies be included? Drug Discovery Today, } & 9(21), \quad 924-931 .\end{array}$ http://dx.doi.org/10.1016/S1359-6446(04)03274-X

Simmonds, M. C., Higgins, J. P. T., Stewart, L. A., Tierney, J. F., Clarke, M. J., \& Thompson, S. G. (2005). Meta-analysis of individual patient data from randomized trials: a review of methods used in practice. Clin Trials, 2, 209-217. Retrieved from http://www.ncbi.nlm.nih.gov/pubmed/16279144

Stewart, L. A., \& Tierney, J. F. (2002). To IPD or not to IPD? Advantages and disadvantages of systematic reviews using individual patient data. Eval Health Prof, 25, 76-97. Retrieved from http://www.ncbi.nlm.nih.gov/pubmed/11868447

Tudur, C., Williamson, P. R., Kahan, S., \& Best, L. Y. (2001). The value of the aggregated data approach to meta-analysis with time-to-event outcomes. $J$ Royal Stat Soc A, 164, 357-370. http://dx.doi.org/10.1111/1467-985X.00207

Turner, R. M., Omar, R. Z., Yang, M., Goldstein, H., \& Thompson, S. G. (2000). A multilevel model framework for meta-analysis of clinical trials with binary outcomes. Stat Med, 9, 3417-3432. Retrieved from http://www.ncbi.nlm.nih.gov/pubmed/11122505

Wang, J. G., Staessen, J. A., Franklin, S. S., Fagard, R., \& Gueyffier, F. (2005). Systolic and diastolic blood pressure lowering as determinants of cardiovascular. Hypertension, 45, 907-913. Retrieved from http://www.ncbi.nlm.nih.gov/pubmed/15837826

Whitehead, A., Omar, R. Z., Higgins, J. P., Savaluny, E., Turner, R. M., \& Thompson, S. G. (2001). Meta-analysis of ordinal outcomes using individual patient data. Stat Med, 20, 2243-2260. Retrieved from http://www.ncbi.nlm.nih.gov/pubmed/11468762

\section{Copyrights}

Copyright for this article is retained by the author(s), with first publication rights granted to the journal.

This is an open-access article distributed under the terms and conditions of the Creative Commons Attribution license (http://creativecommons.org/licenses/by/3.0/). 